\title{
One-ended subgroups of graphs of free groups with cyclic edge groups
}

\author{
HENRY WILTON
}

\begin{abstract}
Consider a one-ended word-hyperbolic group. If it is the fundamental group of a graph of free groups with cyclic edge groups then either it is the fundamental group of a surface or it contains a finitely generated one-ended subgroup of infinite index. As a corollary, the same holds for limit groups. We also obtain a characterisation of surfaces with boundary among free groups equipped with peripheral structures.
\end{abstract}

20F65, 20F67, 57M07

Free subgroups of hyperbolic groups are abundant, and many successful techniques have been developed to find them. It is necessarily much harder to find one-ended subgroups: any one-ended group has at most finitely many conjugacy classes of embeddings into a fixed hyperbolic group; see Gromov [15, 5.3.C']. One case of longstanding interest in topology is the problem of finding a surface subgroup, by which we mean a subgroup isomorphic to the fundamental group of a closed surface of nonpositive curvature. The following famous question is often attributed to Gromov; see the problem lists of Bestvina [4] and Bridson [7].

Question 1 Does every one-ended hyperbolic group have a surface subgroup?

The closest thing to this question that we were able to find in the literature is as follows [15, page 144].

"[O]ne may suspect that there exist word hyperbolic groups $\Gamma$ with arbitrarily large dim $\partial \Gamma$ (here, large is $\geq 1$ ) where every proper subgroup is free."

Setting aside the question of whether every hyperbolic group has a proper subgroup of finite index, which is a notoriously difficult question in its own right, this raises the following natural counterpart to Question 1.

Question 2 Does every one-ended hyperbolic group that is not virtually a surface group contain a finitely generated one-ended subgroup of infinite index? 
Although Question 2 seems substantially weaker than Question 1, in the motivating case of the fundamental group of a closed hyperbolic 3-manifold (recently resolved in the affirmative by Kahn and Markovic [20]), the two questions are equivalent. Indeed, since 3-manifold groups are coherent by Scott [27], any finitely generated, infiniteindex subgroup $H$ of a 3-manifold group is the fundamental group of a compact 3-manifold $N$ with nonempty boundary. If $N$ is aspherical then $\partial N$ has nonpositive Euler characteristic. If, in addition, $H$ is one-ended then Dehn's Lemma implies that $\partial N$ is incompressible, and so $H$, and hence $\pi_{1} N$, has a surface subgroup.

Despite the recent result of Kahn and Markovic, little is known about certain seemingly very simple classes of hyperbolic groups. For instance, let $\Gamma$ be the fundamental group of a graph of free groups with infinite cyclic edge groups. Calegari proved that $\Gamma$ has a surface subgroup if $H_{2}(\Gamma ; \mathbb{Q})$ is nontrivial [9]. Further sufficient conditions were found by Gordon and the author [14] and Kim and the author [23]. Kim and Oum answered Question 1 when $\Gamma$ is the double of a rank-two free group [22].

By the Combination Theorem of Bestvina and Feighn [5], $\Gamma$ is hyperbolic if and only if it does not contain a Baumslag-Solitar subgroup, and the existence of such a subgroup can be verified from a given graph-of-groups decomposition. One-endedness can also be easily characterised in this case: $\Gamma$ is one-ended if and only if every vertex group of the graph of groups is freely indecomposable relative to its edge groups; see Theorem 18 below for details.

The main theorem of this paper resolves Question 2 for such $\Gamma$.

Theorem 3 Let $\Gamma$ be the fundamental group of a graph of free groups with cyclic edge groups. If $\Gamma$ is hyperbolic and one-ended then either $\Gamma$ is the fundamental group of a closed surface or $\Gamma$ has a finitely generated subgroup of infinite index that is one-ended.

It is obvious that one can reduce to the case in which the given splitting of $\Gamma$ has only one edge - that is, to the case in which $\Gamma$ is an amalgamated free product or HNN extension of free groups. However, this observation does not seem to be particularly useful. In fact, the proof uses a cyclic splitting of $\Gamma$ that may be finer, namely the JSJ decomposition.

Of course, graphs of free groups with cyclic edge groups are not representative of hyperbolic groups in general, so Theorem 3 falls far short of resolving Question 2, but it does place heavy restrictions on the nature of any negative example. If $\Gamma$ is any hyperbolic group that splits over a virtually cyclic subgroup then Question 2 is trivial for $\Gamma$ unless $\Gamma$ is the fundamental group of a graph of virtually free groups with virtually cyclic edge groups. Such groups are virtually torsion-free by Wise [33, Theorem 4.19], and so the main theorem has the following corollary. 
Corollary 4 Suppose $\Gamma$ is a one-ended hyperbolic group that splits nontrivially over a virtually cyclic subgroup. Then either $\Gamma$ has a finitely generated one-ended subgroup of infinite index or $\Gamma$ is virtually the fundamental group of a closed surface.

Limit groups, otherwise known as finitely generated fully residually free groups, play a central role in the study of algebraic geometry and logic over free groups; see Sela [29] and Kharlampovich and Myasnikov [21]. They are not necessarily hyperbolic, but every limit group is a toral relatively hyperbolic group; see Alibegović [1] and Dahmani [12].

Corollary 5 If $\Gamma$ is a one-ended limit group then either $\Gamma$ has a finitely generated one-ended subgroup of infinite index or $\Gamma$ is the fundamental group of a closed surface.

Proof A limit group is hyperbolic if and only if it does not have a subgroup isomorphic to $\mathbb{Z}^{2}$ [29, Corollary 4.4], and any virtually abelian limit group is abelian, so we may reduce to the hyperbolic case. Every nonabelian limit group splits over a cyclic subgroup [29, Theorem 3.9], so the result follows from Corollary 4.

Further motivation for Theorem 3 is provided by the class of special groups, introduced by Haglund and Wise [17], of which graphs of free groups with cyclic edge groups are examples by Hsu and Wise [19]. It should be possible to generalise Theorem 3 to the class of special groups; the main technical obstruction is the absence of a suitable JSJ decomposition.

The ingredients of the proof of Theorem 3 include Bowditch's JSJ decomposition for hyperbolic groups and a criterion for detecting free splittings of graphs of groups with cyclic edge groups in terms of their vertex groups (Theorem 18). The heart of the proof is a "Local Theorem" about the rigid vertices of the JSJ decomposition (Theorem 8 below). To state the Local Theorem, we need to introduce peripheral structures on free groups.

A multiword in $F$ is a subset $\underline{w} \subseteq F \backslash 1$. A set of pairwise nonconjugate maximal cyclic subgroups of $F$ is called a peripheral structure on $F$. Any multiword $\underline{w}$ defines a peripheral structure $[\underline{w}]$. We will consider pairs $(F,[\underline{w}])$, where $F$ is a free group and $[\underline{w}]$ is a peripheral structure. Note that any vertex group of a graph of groups with cyclic edge groups is naturally equipped with a peripheral structure induced by the incident edges.

A peripheral structure $[\underline{w}]$ on $F$ induces a natural pullback peripheral structure $[\underline{\hat{w}}]$ on any subgroup $\widehat{F} \subseteq F$. We give a topological definition. 
Definition 6 Realise $F$ as the fundamental group of a handlebody $X$, and $\underline{w}$ as an embedded 1-dimensional submanifold $N \subseteq X$. If $\widehat{F} \subseteq F$ is a subgroup, let $\hat{X}$ be a corresponding covering space of $X$ and let $\hat{N}$ be the preimage of $N$ in $\hat{X}$. The pullback of $[\underline{w}]$ to $\hat{F}$, which we denote by $[\underline{\hat{w}}]$, is determined by those nontrivial conjugacy classes of $\widehat{F}$ that are determined by components of $\hat{N}$.

Remark 7 If $\underline{w}$ is finite and $\widehat{F}$ is finitely generated then $\underline{\hat{w}}$ is finite.

A pair $(F,[\underline{w}])$ is said to be freely indecomposable or one-ended if the elements of $[\underline{w}]$ are hyperbolic in every free splitting of $F$, and rigid if they are hyperbolic in every cyclic splitting of $F$. A pair $(F,[\underline{w}])$ is said to be a surface if there is an isomorphism $F \cong \pi_{1} \Sigma$ for $\Sigma$ a compact surface that identifies $[\underline{w}]$ with the conjugacy classes of cyclic subgroups corresponding to $\partial \Sigma$. An important special case is when $\Sigma$ is a thrice-punctured sphere; this is the unique case in which $(F,[\underline{w}])$ is both a surface and rigid.

We can now state the Local Theorem.

Theorem 8 Suppose $(F,[\underline{w}])$ is rigid and not a thrice-punctured sphere. For any clean finite-index subgroup $\widehat{F} \subseteq F$, for any $\widehat{w}_{i} \in \underline{\widehat{w}}$, the pair $\left(\widehat{F},\left[\underline{\hat{w}} \backslash\left\{\widehat{w}_{i}\right\}\right]\right)$ is freely indecomposable.

See Definition 27 below for the definition of a clean subgroup. It follows from Marshall Hall's Theorem [18] that there are many clean subgroups of finite index.

Finally, let us consider the extent to which the results of this paper have a bearing on Question 1. Our techniques provide a new characterisation of surfaces with boundary $\left(\pi_{1} \Sigma, \partial \Sigma\right)$ among all pairs $(F,[\underline{w}])$. To express this characterisation, we introduce the natural partial order on commensurability classes of subgroups of free groups with peripheral structures. A peripheral structure $[\underline{u}]$ on $\hat{F}$ is compatible with $[\underline{w}]$ if $[\underline{u}] \subseteq[\underline{\hat{w}}]$. We define a preorder on subgroups of $F$ equipped with compatible peripheral structures as follows.

Definition 9 Given $(F,[\underline{w}])$, let $(H,[\underline{u}])$ and $(K,[\underline{v}])$ be subgroups equipped with peripheral structures compatible with $[\underline{w}]$. Let $[\underline{\hat{u}}]$ be the peripheral structure on $H \cap K$ induced by $[u]$. Write

$$
(H,[\underline{u}]) \leq(K,[\underline{v}])
$$

if:

(1) $|H: H \cap K|<\infty$; and furthermore,

(2) if $|K: H \cap K|<\infty$ also then [스] is compatible with [v]]. 
This is a preorder on subgroups equipped with compatible peripheral structures; the induced equivalence relation is called commensurability, and the preorder $\leq$ descends to a partial order on commensurability classes.

Definition 10 Let $\mathcal{P}(\underline{w})$ be the poset of commensurability classes of pairs $(H,[\underline{u}])$ such that:

(1) $H$ is nonabelian and a finitely generated subgroup of $F$;

(2) $[\underline{u}]$ is compatible with $[\underline{w}]$;

(3) the pair $(H,[u])$ is freely indecomposable.

Corollary 11 Suppose that $(F,[\underline{w}])$ is freely indecomposable. Let $(H,[\underline{u}])$ be a pair that represents a commensurability class in $\mathcal{P}(\underline{w})$. The commensurability class represented by $(H,[\underline{u}])$ is minimal in $\mathcal{P}(\underline{w})$ if and only if

$$
(H,[\underline{u}]) \cong\left(\pi_{1} \Sigma, \partial \Sigma\right)
$$

for some compact surface with boundary $\Sigma$.

This raises the hope of applying Zorn's Lemma to find surface subgroups.

Question 12 Does every chain in $\mathcal{P}(\underline{w})$ have a lower bound?

An affirmative answer to Question 12 would come very close to resolving Question 1 in the case of graphs of free groups. (There are also some mild compatibility conditions needed on the surfaces constructed.) This would indicate that surfaces with boundary in $[\underline{w}]$ are fairly abundant in $F$. A negative answer would indicate that such surfaces are very special indeed.

Acknowledgements Thanks to Sang-hyun Kim for pointing me towards Question 2. Thanks to Cameron Gordon for teaching me everything I know about handlebodies and Whitehead's algorithm, and also for a useful conversation about Lemma 22. Thanks to Chris Cashen and Larsen Louder for some instructive examples and to the referee for catching some errors in an earlier draft.

\section{Covering theory of graphs of spaces}

In the proof of Theorem 3, we will need to use the covering theory of graphs of groups. Such a theory was developed from an algebraic point of view by Bass [2], but instead we will use the point of view of graphs of spaces, following Scott and Wall [28]. 
The data for a graph of spaces $X$ are as follows. We are given a graph $\Xi$, for each vertex $v$ of $\Xi$ a connected CW-complex $X_{v}$, and for each edge $e$ of $\Xi$ a connected CWcomplex $X_{e}$. If an edge $e$ adjoins vertices $v_{ \pm}$, we are given corresponding attaching maps $\partial_{e}^{ \pm}: X_{e} \rightarrow X_{v_{ \pm}}$. These attaching maps $\partial_{e}^{ \pm}$are required to be $\pi_{1}$-injections.

The geometric realisation of $X$ is the space

$$
\left(\coprod_{v \in V(\Xi)} X_{v} \sqcup \coprod_{e \in E(\Xi)}\left(X_{e} \times[-1,+1]\right)\right) / \sim
$$

where the relation $\sim$ identifies $(x, \pm 1) \in X_{e} \times[-1,+1]$ with $\partial_{e}^{ \pm}(x) \in X_{v_{ \pm}}$, for each edge $e \in E(\Xi)$ and each $x \in X_{e}$. We will usually abuse notation and denote the geometric realisation of $X$ by $X$.

From this topological point of view, a graph of groups with fundamental group $\Gamma$ is simply an Eilenberg-Mac Lane space $X$ for $\Gamma$ with the structure of a graph of spaces.

The key component of the covering theory for graphs of spaces is the definition of an elevation, which was first introduced by Wise (see, for instance, [33]). The covering theory of graphs of spaces was further developed by the author in $[31 ; 32]$, to which the reader is referred for proofs of some of the statements below.

Let $X$ be a graph of spaces, with underlying graph $\Xi$, vertex spaces $X_{v}$, edge spaces $X_{e}$, and attaching maps $\partial_{e}^{ \pm}: X_{e} \rightarrow X_{v}$. A covering space $\hat{X}$ of $X$ is naturally endowed with the structure of a graph of spaces. The connected components of the preimages of the vertex spaces of $X$ form the vertex spaces of $\hat{X}$, and likewise the connected components of the preimages of the edge cylinders of $X$ form the edge cylinders of $\hat{X}$. The underlying graph of $\hat{X}$, denoted by $\widehat{\Xi}$, can be recovered by collapsing the vertex spaces of $\widehat{X}$ to points and the edge spaces of $\widehat{X}$ to arcs. The covering map $\hat{X} \rightarrow X$ induces a combinatorial map of underlying graphs $\widehat{\Xi} \rightarrow \Xi$, which sends vertices to vertices and edges to edges.

It remains to describe the attaching maps of the covering space $\hat{X}$. Given an attaching map $\partial_{e}^{ \pm}: X_{e} \rightarrow X_{v}$ of $X$ and a vertex space $\hat{X}_{\hat{v}}$ of $\hat{X}$, the disjoint union of the edge spaces $\left\{\hat{X}_{\hat{e}}\right\}$ of $\hat{X}$ that lie above $X_{e}$, together with the coproduct of their attaching maps, fits into a commutative diagram as follows.

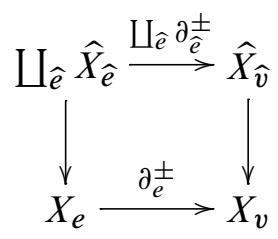


Here, $\widehat{e}$ ranges over all the edges of $\widehat{\Xi}$ in the preimage of $e$ that are incident with $\widehat{v}$. The key observation is that this diagram is a pullback. The restriction of the pullback of a continuous map and a covering map to a connected component is called an elevation. See $[31 ; 32 ; 33]$ for further characterisations of elevations. Therefore, the attaching maps of $\hat{X}$ are precisely the elevations of the attaching maps of $X$ to the vertex spaces of $\hat{X}$. In particular, the covering space $\hat{X}$ and covering map $\hat{X} \rightarrow X$ are determined by the restriction of the covering map to the vertex spaces and by the map of graphs $\widehat{\Xi} \rightarrow \Xi$.

There is a condition on a set of covering maps of the vertex spaces that determines whether or not they can be extended to a covering of $X$. The degree of an elevation is the conjugacy class of $\pi_{1}\left(\hat{X}_{\widehat{e}}\right)$ as a subgroup of $\pi_{1}\left(X_{e}\right)$.

Proposition 13 Let $\left\{\widehat{X}_{\widehat{v}} \rightarrow X_{v}\right\}$ be a set of covering maps of the vertex spaces $\left\{X_{v}\right\}$ of a graph of spaces $X$. These covering maps can be extended to a covering map $\hat{X} \rightarrow X$, where the $\left\{\hat{X}_{\hat{v}}\right\}$ are the vertex spaces of $\hat{X}$ in the induced graph-of-spaces decomposition, if and only if the following condition holds. For each edge $e$ of $\Xi$ with attaching maps $\partial_{e}^{ \pm}: X_{e} \rightarrow X_{v_{ \pm}}$, there is a degree-preserving bijection between the set of elevations of $\partial_{e}^{+}$to $\bigsqcup_{\widehat{v}} \hat{X}_{\widehat{v}}$ and the set of elevations of $\partial_{e}^{-}$to $\bigsqcup_{\widehat{v}} \hat{X}_{\widehat{v}}$.

More generally, any degree-preserving bijection between subsets of the sets of elevations of the edge maps of $X$ to $\bigsqcup_{v^{\prime}} X_{v^{\prime}}^{\prime}$ can be used to build a graph of spaces $X^{\prime}$ with vertex spaces $\left\{X_{v^{\prime}}^{\prime}\right\}$ and a map $X^{\prime} \rightarrow X$. The resulting space $X^{\prime}$ is called a precover of $X$. The elevations to $X^{\prime}$ of attaching maps of $X$ that are not attaching maps of $X^{\prime}$ are called hanging elevations. The previous proposition can be generalised as follows.

Proposition 14 A precover $X^{\prime}$ of a graph of spaces $X$ can be extended to a covering map $\hat{X} \rightarrow X$, where every vertex space of $\hat{X}$ is a vertex space of $X^{\prime}$, if and only if the following condition holds. For each edge $e$ of $\Xi$ with attaching maps $\partial_{e}^{ \pm}: X_{e} \rightarrow X_{v_{ \pm}}$, there is a degree-preserving bijection between the set of hanging elevations of $\partial_{e}^{+}$to $X^{\prime}$ and the set of hanging elevations of $\partial_{e}^{-}$to $X^{\prime}$.

It follows that precovers can always be extended to covers.

Lemma 15 If $X^{\prime}$ is a precover of $X$ then the map $X^{\prime} \rightarrow X$ can be extended to a covering map $\hat{X} \rightarrow X$, where $X^{\prime}$ is a subgraph of spaces of $\hat{X}$ and the inclusion $X^{\prime} \hookrightarrow \widehat{X}$ induces an isomorphism on fundamental groups.

Proof Let $\partial_{\widehat{e}}^{ \pm}: \hat{X}_{\widehat{e}} \rightarrow X^{\prime}$ be a hanging elevation to $X^{\prime}$ of an attaching map $\partial_{e}^{ \pm}: X_{e} \rightarrow X_{v}$. For each such elevation $\partial_{\widehat{e}}^{ \pm}$, let $Y_{\widehat{e}}$ be the covering space of $X$ with fundamental 
group $\pi_{1}\left(\widehat{X}_{\widehat{e}}\right)$. There is an edge space of $Y_{\widehat{e}}$ with fundamental group $\pi_{1}\left(\widehat{X}_{\widehat{e}}\right)$. Delete this edge space from $Y_{\widehat{e}}$, and let $Z_{\widehat{e}}$ be a component of the result. Then $Z_{\widehat{e}}$ is a precover of $X$ with a unique hanging elevation, which we denote by $\partial_{\widehat{e}}^{\mp}: \hat{X}_{\widehat{e}} \rightarrow Z_{\widehat{e}}$. The precover of $X$ that consists of the disjoint union of $X^{\prime}$ and all the $Z_{\widehat{e}}$, where $\widehat{e}$ ranges over all the hanging elevations to $X^{\prime}$ of attaching maps of $X$, satisfies the hypotheses of the previous proposition. The resulting covering space $\hat{X}$ has the required properties by construction.

As an immediate result, precovers define subgroups.

Lemma 16 If $X^{\prime}$ is a connected precover of a graph of spaces $X$ then the map $X^{\prime} \rightarrow X$ induces a monomorphism at the level of fundamental groups.

A graph of spaces $X$ is called reduced if no attaching map is a $\pi_{1}-$ surjection.

Lemma 17 Suppose $X$ is a reduced graph of spaces. If $X^{\prime}$ is a precover but not a cover of $X$ then $\pi_{1}\left(X^{\prime}\right)$ has infinite index in $\pi_{1}(X)$.

Proof Consider the space $Y_{\widehat{e}}$ constructed in the proof of Lemma 15, and let $\Upsilon$ be its underlying graph. Because $\pi_{1}\left(Y_{\hat{e}}\right)$ is equal to the fundamental group of one of its edge spaces, $\Upsilon$ is a tree. Because $X$ is reduced, every vertex of $\Upsilon$ has valence greater than one. Therefore, $\Upsilon$ is infinite. It follows that there are points of $X$ with infinitely many preimages in $Z_{\widehat{e}}$, and hence $\hat{X} \rightarrow X$ has infinite degree.

\section{A variant of a theorem of Shenitzer}

In this section, we prove a theorem that describes when the fundamental group of a graph of groups with cyclic edge groups splits freely.

Theorem 18 Let $\Gamma$ be finitely generated, and the fundamental group of a graph of groups with infinite cyclic edge groups. Then $\Gamma$ is one-ended if and only if every vertex group is freely indecomposable relative to the incident edge groups.

This statement is similar to a theorem of Shenitzer [30], which says that an amalgam of two free groups along a cyclic group is free if and only if the amalgamating cyclic group is a free factor in one of the free groups-see Louder [24] for a modern treatment and a generalisation. See also Diao and Feighn [13]. In the case of doubles, Theorem 18 was stated without proof in Gordon and Wilton [14]. It is undoubtedly well known to experts, but we were unable to find a proof in the literature, so we give one here. 
Remark 19 The hypothesis that the edge groups are cyclic cannot be removed. Indeed, the free group of rank two can be written as an HNN extension of $F_{3} \cong\langle a, b, c\rangle$ that conjugates $\langle a, b\rangle$ to $\langle b, c\rangle$, but $F_{3}$ does not split freely relative to $\langle a, b\rangle$ and $\langle b, c\rangle$.

One direction of the theorem is obvious: if some vertex group splits freely relative to the incident edge groups then $\Gamma$ also splits freely.

To prove the other direction, we will realise $\Gamma$ as the fundamental group of a certain complex of groups; see Bridson and Haefliger [8] for a detailed discussion of Haefliger's theory of complexes of groups. By hypothesis, we are given a splitting of $\Gamma$ with finitely generated vertex groups $\Gamma_{v}$ and infinite cyclic edge groups $\Gamma_{e}$; let $\Xi$ be the underlying graph of this splitting. Now let $T$ be the Bass-Serre tree of a nontrivial free splitting of $\Gamma$. Because the edge stabilisers of $T$ are trivial, any finitely generated nontrivial subgroup $H$ of $\Gamma$ has a unique minimal invariant subtree of $T$, on which $H$ acts cocompactly. Let $T_{v}$ be the minimal invariant subtree of the vertex group $\Gamma_{v}$, and write $X_{v}$ for the quotient graph of groups $T_{v} / \Gamma_{v}$. Likewise, let $T_{e}$ be the minimal invariant subtree of the edge group $\Gamma_{e}$, which is either a point or a line; again, write $X_{e}$ for the quotient graph of groups $T_{e} / \Gamma_{e}$, which is either topologically a circle or a point labelled by $\Gamma_{e}$. The graphs of groups $X_{v}$ and $X_{e}$ are finite. If $e$ is incident at $v$ then there is a natural inclusion $T_{e} \rightarrow T_{v}$ which descends to a morphism of graphs of groups $X_{e} \rightarrow X_{v}$.

Using these morphisms $X_{e} \rightarrow X_{v}$ as attaching maps, we can build a complex of groups, indeed a graph of graphs of groups, $\mathcal{X}$, with fundamental group $\Gamma$, just as we built a graph of spaces at the beginning of Section 1. That is, the vertex "spaces" of $\mathcal{X}$ are the graphs of groups $X_{v}$, the edge "spaces" are the cylinders $X_{e} \times[-1,1]$, the attaching maps are given by the morphisms $X_{e} \rightarrow X_{v}$, and the underlying graph is $\Xi$. The universal cover of $\mathcal{X}$, which we denote by $\widetilde{\mathcal{X}}$, is a tree of copies of trees $T_{v}$, glued along strips or arcs of the form $T_{e} \times[-1,1]$. The underlying tree of $\tilde{\mathcal{X}}$, which is the Bass-Serre tree of the given cyclic splitting of $\Gamma$, we denote by $\mathcal{T}$.

There is a natural $\Gamma$-equivariant map $f: \tilde{\mathcal{X}} \rightarrow T$ defined as follows: the restriction of $f$ to $T_{v}$ is the inclusion $T_{v} \hookrightarrow T$; on $T_{e} \times[-1,+1], f$ is the composition of the projection to $T_{e}$ with the inclusion $T_{e} \hookrightarrow T$. Let $t$ be the midpoint of an edge in $T$, let $\tilde{Y}_{t}=f^{-1}(t)$, and let $Y_{t}$ be the image of $\tilde{Y}_{t}$ in $\mathcal{X}$. By construction, for any $v$ the intersection of $Y_{t}$ with $X_{v}$ is a finite union of points, and the intersection of $Y_{t}$ with $X_{e} \times[-1,+1]$ is a finite union of arcs of the form $* \times[-1,+1]$. Therefore $Y_{t}$ is topologically a finite graph, with vertices in the vertex graphs of groups of $\mathcal{X}$ and edges in the edge cylinders of $\mathcal{X}$.

Lemma 20 The inclusion $Y_{t} \hookrightarrow \mathcal{X}$, when restricted to a component, induces an injection on fundamental groups. 
Proof Let $Z$ be a component of $Y_{t}$ and let $\tilde{Z}$ be a component of the preimage of $Z$ in $\tilde{\mathcal{X}}$. The lemma follows from the fact that the composition $\widetilde{Z} \hookrightarrow \tilde{\mathcal{X}} \rightarrow \mathcal{T}$ is injective. Suppose not. Then, because $\mathcal{T}$ is a tree, some pair of adjacent edges in $\widetilde{Z}$ map to the same edge in $\mathcal{T}$. This implies that this pair of edges of $\widetilde{Z}$ is contained in a single edge strip $T_{e} \times[-1,+1]$ of $\tilde{\mathcal{X}}$. But the gluing maps map $T_{e} \rightarrow T_{v}$ are injective, so no two edges of $\tilde{Z}$ contained in $T_{e} \times[-1,+1]$ are adjacent.

Proof of Theorem 18 The free splitting of $\Gamma$ represented by $T$ is assumed to be nontrivial, so there is an edge of $T$ in the image of $\tilde{\mathcal{X}}$; let $t$ be the midpoint of this edge. Because the map $\tilde{Y}_{t} \rightarrow T$ is $\Gamma$-equivariant and its image is a point, any loop in $Y_{t}$ stabilises an edge in $T$. But the splitting corresponding to $T$ has trivial edge groups, so $Y_{t}$ is a finite forest. Therefore, there is a vertex graph of groups $X_{v}$ with an edge $\epsilon$ whose midpoint is either an isolated vertex or a leaf in $Y_{t}$. In the former case, deleting the midpoint of $\epsilon$ splits $\Gamma_{v}$ freely, relative to its incident edge groups. In the latter case, there is a unique edge $e$ incident at $v$ with $\epsilon$ in the image of $X_{e}$, and only one edge of $X_{e}$ maps to $\epsilon$. It follows that $\epsilon$ is nonseparating in $X_{v}$-if it were separating, then every loop in $X_{v}$ would cross it an even number of times. Therefore, $\epsilon$ corresponds to a basis element of $\Gamma_{v}$; because $\epsilon$ is crossed once by $X_{e}$ and is not crossed by the other incident edge spaces, there is a splitting of $\Gamma_{v}$ relative to the incident edge groups.

\section{Whitehead graphs}

The aim of this section is to prove Theorem 8. To do this, we first need to recall something about Whitehead's algorithm. We will adopt the discs-in-handlebodies point of view_-see Berge [3] and Manning [26] for clear accounts. For the combinatorial perspective on Whitehead's algorithm, see for instance Lyndon and Schupp [25].

Let $U$ be a handlebody of appropriate genus, and fix an identification $F \cong \pi_{1}(U)$. The conjugacy classes of the elements of a multiword $\underline{w}$ naturally correspond to a 1-dimensional submanifold $N \subseteq U$, determined up to homotopy, in which each component is essential. Any choice of basis $B$ for $F$ naturally corresponds to a maximal family of separating discs $\mathcal{D} \subseteq U$; when $U$ is cut along $\mathcal{D}$, the result is a 3-ball; the preimage of each disc is a pair of discs on the boundary of the ball, and the preimage of $N$ is a union of arcs in the ball, with their endpoints in the discs. Crushing the discs to points, the resulting 1-complex embedded in the ball is the Whitehead graph of $\underline{w}$, with respect to $B$. It is convenient to remember the pairing on the vertices. We will denote the Whitehead graph of a multiword $\underline{w}$ with respect to $B$ by $W_{B}(\underline{w})$. 


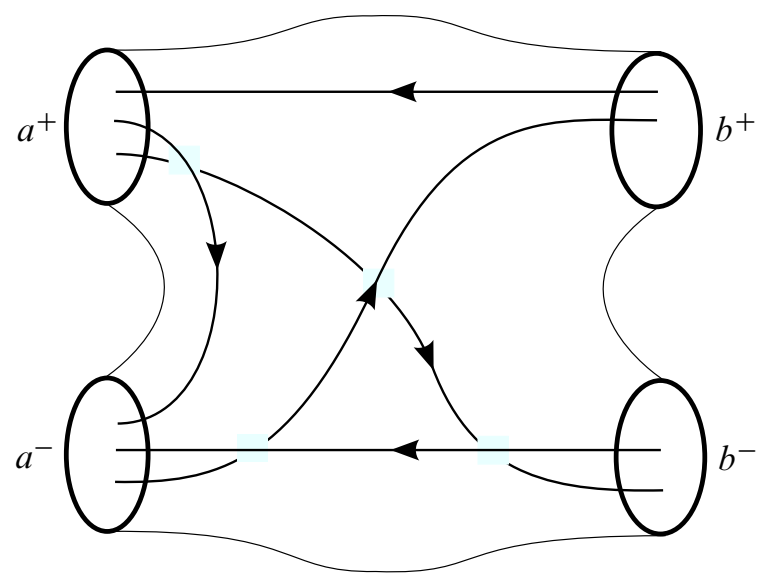

Figure 1. The word $b a^{-1} b^{-1} a^{2}$ realised as a submanifold of a handlebody, which is cut along the discs that represent the standard generating set

We will often suppress all mention of $B$ when it causes no confusion. We will call a multiword $\underline{w}$ minimal if the basis $B$ is chosen to minimise its length.

If there is a pair of vertices $\left\{v^{+}, v^{-}\right\}$of $W_{B}(w)$ separated by a set $\mathcal{E}$ of edges of $W_{B}(\underline{w})$, and the cardinality of $\mathcal{E}$ is strictly less than the valence of $v^{+}$(which equals the valence of $v^{-}$) then a Whitehead move can be applied, which yields a new basis $B^{\prime}$ such that $W_{B^{\prime}}(\underline{w})$ has fewer edges than $W_{B}(\underline{w})$.

For our purposes, Whitehead graphs will be useful for recognising free and cyclic splittings of $(F,[\underline{w}])$. The following lemma is standard—see, for instance, Cashen [11, Theorem 4.1].

Lemma 21 If $W(\underline{w})$ is disconnected then $(F, \underline{w})$ is freely decomposable. Conversely, if $(F, \underline{w})$ is freely decomposable then $W(\underline{w})$ is either disconnected or contains a separating vertex.

Note that if $W(\underline{w})$ contains a separating vertex then $\underline{w}$ is not minimal. Next, we need a condition to recognise rigid pairs. The following result follows from work of Cashen [10], but we shall give a more direct proof here.

Lemma 22 If $W(\underline{w})$ contains a separating pair of edges then $(F,[\underline{w}])$ is either a thrice-punctured sphere or is not rigid.

Proof As above, we realise $\underline{w}$ by an embedded 1-dimensional submanifold in a handlebody $U$. We will abuse notation and denote by $w_{i}$ the component of the submanifold corresponding to the element $w_{i} \in \underline{w}$. Let $w_{i}, w_{j} \in \underline{w}$ be the (not necessarily 
distinct) components of $\underline{w}$ that contain the separating pair of edges. By hypothesis, there exists a disc $D$ properly embedded in $U$ such that $w_{i} \cup w_{j}$ intersects $D$ in exactly two points and every other $w_{k}$ is disjoint from $D$. Let $V$ be a small closed neighbourhood of $D \cup w_{i} \cup w_{j}$, let $\Sigma$ be the boundary of $V$ in $U$ and let $V^{\prime}$ be the complement of the interior of $V$.

Fix a basepoint in $\Sigma$. There are various cases to consider, according to whether or not $w_{i}$ and $w_{j}$ are distinct and whether or not $\Sigma$ is connected. We will assume that they are distinct and that $\Sigma$ is connected, and leave the remaining cases to the reader.

By construction, $\pi_{1}(V) \cong\left\langle w_{i}\right\rangle *\left\langle w_{j}\right\rangle$ (choosing representatives of conjugacy classes appropriately). The surface $\Sigma$ is homeomorphic to a twice-punctured torus, and its fundamental group can be presented as

$$
\pi_{1}(\Sigma) \cong\left\langle a, b, d_{1}, d_{2} \mid[a, b] d_{1} d_{2}\right\rangle
$$

where $a=w_{i} w_{j}, b$ is a meridian about $w_{i}$, and $d_{1}$ and $d_{2}$ are freely homotopic to the boundary components of $\Sigma$. The Seifert-van Kampen Theorem implies that $F \cong \pi_{1}(U)$ is a pushout.

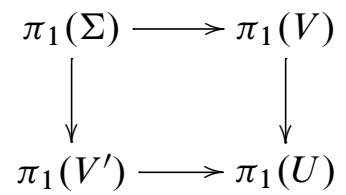

Factoring out the kernels of the maps to $\pi_{1}(U)$, we obtain a decomposition of $\pi_{1}(U)$ as an amalgamated free product. Because $b, d_{1}$ and $d_{2}$ are all null-homotopic in $U$, whereas $a$ survives, the edge group is cyclic.

If this cyclic splitting is trivial then necessarily $w_{i}$ and $w_{j}$ generate $F$, and then either $(F,[\underline{w}])$ is freely decomposable or

$$
[\underline{w}]=\left[\left\{w_{i}, w_{j}, w_{i} w_{j}\right\}\right],
$$

in which case $(F,[\underline{w}])$ is a thrice-punctured sphere.

Lemma 23 If $(F,[\underline{w}])$ is rigid and $W(\underline{w})$ contains a vertex $v$ and an edge $e$ such that $W(\underline{w}) \backslash\{v, e\}$ is disconnected then either $(F,[\underline{w}])$ is a thrice-punctured sphere or $\underline{w}$ is not minimal.

Proof Let $A, B$ be the components of $W(\underline{w}) \backslash\{v, e\}$. Without loss of generality, assume that $u$, the pair of $v$, is contained in $A$. Let $E_{A}$ and $E_{B}$ be the sets of edges that joins $v$ to $A$ and $B$ respectively. Then $E_{A} \cup\{e\}$ is a set of edges that separates $v$ from $u$, so one can perform a Whitehead move, and hence $\underline{w}$ is not minimal, unless 
the valence of $v$ is equal to the cardinality of $E_{A} \cup\{e\}$. But this is true if and only if $E_{B}$ is a single edge $f$, in which case $\{e, f\}$ is a separating pair of edges.

These conditions are certainly not exhaustive. There are cyclically decomposable pairs for which the minimal Whitehead graph does not contain a cut pair of edges.

Next, we need to understand the Whitehead graphs of pullbacks. The key operation is splicing, which was introduced by Manning [26].

Definition 24 Let $A, B$ be finite graphs. Let $u$ be a vertex of $A$ and $v$ a vertex of $B$, and assume that the valences of $u$ and $v$ are equal. Fix a bijection $f$ between the edges of $A$ incident at $u$ and the edges of $B$ incident at $v$. Construct a new graph $C$ as follows: delete the vertices $u$ and $v$ from $A$ and $B$, leaving the incident edges "hanging"; then glue the resulting hanging edges of $A$ and $B$ together according to the bijection $f$. The graph $C$ is said to be obtained from $A$ and $B$ by splicing.

The construction of the Whitehead graph of a pullback can be summarised in the following lemma. An example is illustrated in Figure 2.

Lemma 25 (Manning [26]) If $\widehat{F} \subseteq F$ is a subgroup of finite index and $\underline{w}$ is the pullback of $\underline{w}$ to $\widehat{F}$ then $W(\underline{\hat{w}})$ is obtained by splicing $|F: \widehat{F}|$ copies of $W(\underline{w})$. (The choices made when splicing correspond exactly to the choice of basis for $\hat{F}$.)

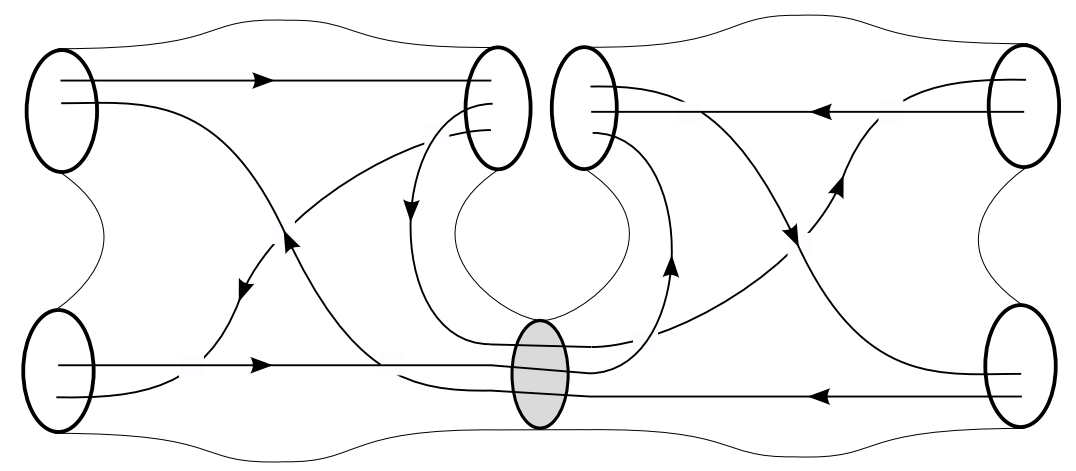

Figure 2. The lift of $b a^{-1} b^{-1} a^{2}$ to a cover of degree two. Deleting the grey disc corresponds to splicing.

Remark 26 The result of splicing two connected graphs without cut vertices is another connected graph without cut vertices. The proof of this is left as an exercise to the reader. 
The final piece of the argument is the notion of a clean cover (or, equivalently, a clean subgroup), used extensively by Wise.

Definition 27 Fix a basis $B$ for $F$ and consider a pair $(F,[\underline{w}])$. Let $X$ be the rose graph, oriented and labelled, with the identification $F \cong \pi_{1}(X)$ determined by the choice of basis $B$. Consider a subgroup $\widehat{F}$ of finite index in $F$, and the corresponding covering map $\hat{X} \rightarrow X$. The subgroup $\hat{F}$ is called clean if the pullback map

$$
\underline{\widehat{w}}: \coprod_{i} S^{1} \rightarrow \hat{X}
$$

is injective on each connected component.

In the handlebody picture, the subgroup $\widehat{F}$ is clean if and only if every component of the preimage of $\underline{w}$ intersects each ball in at most one arc. See Figure 3.

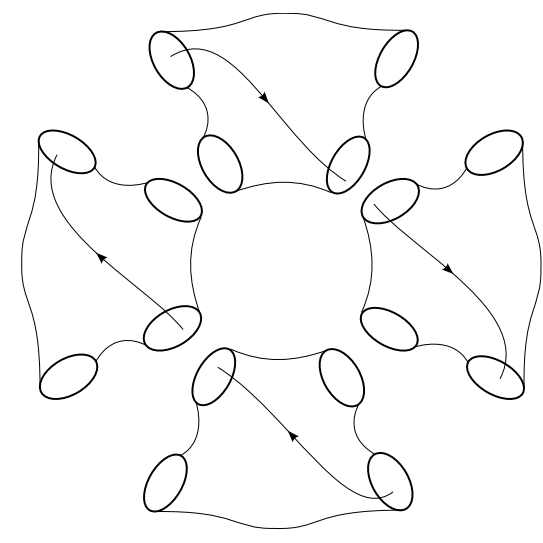

Figure 3. One component of the pullback of a word to a clean cover

A standard application of Marshall Hall's Theorem shows that clean subgroups are plentiful.

Lemma 28 If $\bar{F}$ is any subgroup of finite index in $F$ then there is a clean subgroup $\widehat{F} \subseteq \bar{F}$.

Proof For each element $w_{i}$ of $\underline{w}$, Marshall Hall's Theorem provides a finite-index subgroup $F_{i} \subseteq F$ such that, if $X_{i} \rightarrow X$ is the corresponding finite-sheeted cover, the map $w_{i}: S^{1} \rightarrow X$ lifts to an embedding $S^{1} \hookrightarrow X_{i}$. The normal core of the subgroup

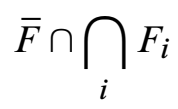

is the required clean subgroup of $F$. 
Proof of Theorem 8 Choose a basis $B$ for $F$ so that $\underline{w}$ is minimal. By Lemma 23 and the hypothesis that $(F, \underline{w})$ is rigid and not a thrice-punctured sphere, the Whitehead graph $W(\underline{w})$ does not contain a separating vertex-edge pair. As described above, the Whitehead graph $W(\underline{\hat{w}})$ is obtained by splicing together various copies $W_{j}$ of $W(\underline{w})$. Because $\widehat{F}$ is clean, $\widehat{w}_{i}$ intersects each $W_{j}$ in at most one edge. For each $j$, let $W_{j}^{\prime}=W_{j} \backslash \widehat{w}_{i}$; note that each $W_{j}^{\prime}$ is connected and does not have a cut vertex. The Whitehead graph $W\left(\underline{\hat{w}} \backslash\left\{\hat{w}_{i}\right\}\right)$ is obtained by splicing together the $W_{j}^{\prime}$, and therefore is also connected with no cut vertices. In particular, $\left(\hat{F},\left[\underline{w} \backslash\left\{\hat{w}_{i}\right\}\right]\right)$ is one-ended by Lemma 21 .

\section{The JSJ decomposition}

To prove the main theorem, we will use the (cyclic) JSJ decomposition of $\Gamma$. JSJ decompositions of groups come in many different versions; Guirardel and Levitt [16] contains a useful summary and a unifying perspective. To be specific, we will use Bowditch's JSJ decomposition [6], which is only defined for hyperbolic groups but has the advantage of being canonical. We summarise its properties in the case of interest to us in the following result.

Theorem 29 (Bowditch [6]) Let $G$ be any one-ended, torsion-free, hyperbolic group. There is a reduced graph of groups, with fundamental group $G$, with the following properties.

(1) Each edge group is cyclic.

(2) Each vertex group is of one of three sorts.

(a) Cyclic vertex groups are cyclic.

(b) Surface vertex groups are isomorphic to the fundamental groups of compact surfaces. The incident edge groups are identified with (powers of) boundary components.

(c) Rigid vertex groups do not split over any cyclic subgroup relative to the incident edge groups.

Furthermore, one end of every edge adjoins a cyclic vertex.

This graph of groups is called the JSJ decomposition of $G$.

Of course, in the above definition there is some ambiguity, as any vertex corresponding to a thrice-punctured sphere can be thought of as either of surface or rigid type. We shall always think of them as being of surface type. 
Remark 30 If $\Gamma$ is the fundamental group of a graph of free groups with cyclic edge groups then the JSJ decomposition of $\Gamma$ is trivial if and only if $\Gamma$ is the fundamental group of a surface.

First, we shall deal with the case in which there are no rigid vertices. The proof of the following lemma is similar to the proof of [14, Lemma 22].

Lemma 31 Suppose the JSJ decomposition of $\Gamma$ has no rigid vertices. Then $\Gamma$ has a surface subgroup. In particular, $\Gamma$ either has a one-ended subgroup of infinite index or $\Gamma$ is a surface group.

Proof By [33, Theorem 4.18], we can pass to a finite-index subgroup $\Gamma_{0}$ of $\Gamma$ and assume that every attaching map identifies a cyclic vertex group with a boundary component of a surface component. It is easy to thicken this picture to see that $\Gamma_{0}$ is the fundamental group of a 3-manifold with boundary, $M$. Standard gluing arguments show that $M$ is aspherical, and it follows that $\chi(\partial M) \leq 0$. By Stallings's Ends Theorem, $\Gamma_{0}$ is one-ended, and so $\partial M$ is incompressible in $M$ by the Loop Theorem and Dehn's Lemma. Therefore $\pi_{1}(\partial M)$ is a surface subgroup of $\Gamma$.

Proof of Theorem 3 Realise the JSJ decomposition of $\Gamma$ by a graph of spaces $X$. By Lemma 31, we may assume that some vertex $v$ of $X$ is rigid, and not a thrice-punctured sphere. That is, if $F=\pi_{1}\left(X_{v}\right)$ and $[\underline{w}]$ is the peripheral structure induced by the incident edge groups, then $(F,[\underline{w}])$ is rigid. Let $\widehat{F}$ be a clean subgroup of finite index in $F$, equipped with the pullback peripheral structure $[\hat{\underline{w}}]$. Because $\Gamma$ is subgroup separable [33], there is a finite-sheeted covering space $\hat{X}$ of $X$, with a vertex space $\hat{x}_{\hat{v}}$ covering $X_{v}$, such that $\pi_{1}\left(\hat{X}_{\widehat{v}}\right)=\widehat{F}$. Let $\widehat{\Gamma}=\pi_{1}(\hat{X})$ and let $\widehat{\Xi}$ be the underlying graph of $\hat{X}$. Let $X^{\prime}$ be the precover of $X$ that consists of the union of every vertex space of $\hat{X}$ apart from $\hat{X}_{\widehat{v}}$, and every edge cylinder of $\hat{X}$ that does not adjoin $\hat{X}_{\widehat{v}}$. Let $e_{1}, \ldots, e_{m}$ be the edges of $\widehat{\Xi}$ with one end adjoining $\hat{v}$, and let $f_{1}, \ldots, f_{n}$ be the edges of $\widehat{\Xi}$ with both ends adjoining $\hat{v}$. We denote by $\partial_{e_{i}}^{+}$the attaching map of $e_{i}$ that maps to $X_{\widehat{v}}$ and by $\partial_{e_{i}}^{-}$the attaching map that maps to $X^{\prime}$. Both $\partial_{f_{j}}^{+}$and $\partial_{f_{j}}^{-}$map to $X_{\widehat{v}}$.

We will now define a new precover $W$ of $\hat{X}$ as follows. Take $m$ copies of $\hat{X}_{\widehat{v}}$, denoted by $Y_{i}$, and take $2 n$ further copies of $\hat{X}_{\hat{v}}$, denoted by $Y_{j}^{ \pm}$. Consider the following subset $\mathcal{E}$ of the set of all elevations of edge maps of $X$ to $Y=\bigsqcup_{i} Y_{i} \sqcup \bigsqcup_{j} Y_{j}^{+} \sqcup \bigsqcup_{j} Y_{j}^{-}$: $\mathcal{E}$ consists of all such elevations, except for the copy of $\partial_{e_{i}}^{+}$that maps to $Y_{i}$, the copy of $\partial_{f_{j}}^{+}$that maps to $Y_{j}^{+}$and the copy of $\partial_{f_{j}}^{-}$that maps to $Y_{j}^{-}$. Each attaching map incident at $\hat{v}$ has exactly $m+2 n-1$ elevations in $\mathcal{E}$, and each of these elevations is a lift (meaning that it is of maximal possible degree). 
Now take $m+2 n-1$ copies of $X^{\prime}$, denoted denote by $Z_{k}$, and let $Z=\bigsqcup_{k} Z_{k}$. The complete set of elevations of edge maps of $\hat{X}$ to $Z$, which we denote by $\mathcal{F}$, consists of exactly $m+2 n-1$ elevations of each $\partial_{e_{i}}^{-}$, and again each of these elevations is a lift. Therefore, $Y \sqcup Z$, together with the elevations $\mathcal{E} \cup \mathcal{F}$, satisfies the hypotheses of Proposition 14, and can be completed to a precover $W$ of $\hat{X}$. If $W$ is disconnected, replace it with one of its connected components. Each vertex group of $W$ is freely indecomposable relative to its incident edge groups, so $\pi_{1}(W)$ is one-ended. The $Y_{i}$ have hanging elevations of edge groups of $\hat{X}$, so $W$ is not a cover of $\hat{X}$. Therefore $\pi_{1}(W)$ is a subgroup of infinite index in $\widehat{\Gamma}$, and hence in $\Gamma$ as required.

Finally, we prove Corollary 11. One possible proof is identical to the proof of Theorem 3, but uses a relative version of the JSJ decomposition, such as that provided by [10]. The proof given here deduces Corollary 11 directly from Theorem 3 by considering doubles.

Given a pair $(F,[\underline{w}])$, the corresponding double $D(F,[\underline{w}])$ is the fundamental group of a graph of groups with two vertices and $\#[\underline{w}]$ edges; each vertex is labelled by a copy of $F$, each edge group is an element of $[\underline{w}]$, and the attaching maps are the natural inclusions. Theorem 18 implies that the pair $(F,[\underline{w}])$ is one-ended if and only if the double $D(F,[\underline{w}])$ is one-ended. Clearly, $D(F,[\underline{w}])$ is a surface group if and only the pair $(F,[\underline{w}])$ can be realised as $\left(\pi_{1} \Sigma, \partial \Sigma\right)$ for some compact surface $\Sigma$.

Proof of Corollary 11 It is clear that a pair of the form $\left(\pi_{1} \Sigma, \partial \Sigma\right)$ is minimal. Conversely, suppose that $(F,[\underline{w}])$ cannot be realised by a surface. Then $D(F,[\underline{w}])$ is not a surface group and so, by Theorem 3, has a finitely generated, one-ended subgroup $H$ of infinite index. Let $X$ be a graph of spaces that realises the given decomposition of $D(F,[\underline{w}])$, and let $X^{H}$ be the covering space corresponding to $H$. Because $H$ is finitely generated, there is a finite subgraph of spaces $X^{\prime} \subseteq X^{H}$ with fundamental group $H ; X^{\prime}$ is naturally a precover of $X$. By Theorem 18, each vertex group of $X^{\prime}$, equipped with the induced peripheral structure, is one-ended. If every vertex space $X_{v^{\prime}}^{\prime}$ of $X^{\prime}$ finitely covered the corresponding vertex space $X_{v}$ of $X$, and if the induced peripheral structure on $\pi_{1} X_{v^{\prime}}^{\prime}$ were always the full pullback of the peripheral structure on $\pi_{1} X_{v}$, then it would follow from the covering theory of graphs of spaces that $X^{\prime} \rightarrow X$ was a finite-sheeted covering map, which would contradict the fact that $H$ is of infinite index in $D(F,[\underline{w}])$. Therefore, there is a vertex space $\pi_{1} X_{v^{\prime}}^{\prime}$ that is either of infinite index in $F$ or such that the peripheral structure $[\underline{u}]$ on $\pi_{1} X_{v^{\prime}}^{\prime}$ induced by $X^{\prime}$ is strictly contained in the pullback peripheral structure $[\underline{\hat{w}}]$. Therefore, $\left(\pi_{1} X_{v^{\prime}}^{\prime},[\underline{u}]\right)<(F,[\underline{w}])$ and so $(F,[\underline{w}])$ is not minimal. 


\section{References}

[1] E Alibegović, A combination theorem for relatively hyperbolic groups, Bull. London Math. Soc. 37 (2005) 459-466 MR2131400

[2] H Bass, Covering theory for graphs of groups, J. Pure Appl. Algebra 89 (1993) 3-47 MR1239551

[3] J Berge, Heegaard documentation (2010) Available at http://www.math.uic.edu/ $\sim \mathrm{t} 3 \mathrm{~m}$

[4] M Bestvina, Questions in geometric group theory, preprint (2004) Available at http://www.math.utah.edu/ bestvina/eprints/questions-updated.pdf

[5] M Bestvina, M Feighn, A combination theorem for negatively curved groups, J. Differential Geom. 35 (1992) 85-101 MR1152226

[6] B H Bowditch, Cut points and canonical splittings of hyperbolic groups, Acta Math. 180 (1998) 145-186 MR1638764

[7] M R Bridson, Problems concerning hyperbolic and CAT(0) groups, preprint (2007) Available at http://aimath.org/pggt/Hyperbolic_and_CAT (0)_Groups

[8] M R Bridson, A Haefliger, Metric spaces of non-positive curvature, Grundl. Math. Wissen. 319, Springer, Berlin (1999) MR1744486

[9] D Calegari, Surface subgroups from homology, Geom. Topol. 12 (2008) 1995-2007 MR2431013

[10] C H Cashen, Splitting line patterns in free groups arXiv:1009.2492v2

[11] C H Cashen, N Macura, Line patterns in free groups, Geom. Topol. 15 (2011) 14191475 MR2825316

[12] F Dahmani, Combination of convergence groups, Geom. Topol. 7 (2003) 933-963 MR2026551

[13] G-A Diao, M Feighn, The Grushko decomposition of a finite graph of finite rank free groups: an algorithm, Geom. Topol. 9 (2005) 1835-1880 MR2175158

[14] C Gordon, H Wilton, On surface subgroups of doubles of free groups, J. Lond. Math. Soc. 82 (2010) 17-31 MR2669638

[15] M Gromov, Hyperbolic groups, from: "Essays in group theory", (S M Gersten, editor), Math. Sci. Res. Inst. Publ. 8, Springer, New York (1987) 75-263 MR919829

[16] V Guirardel, G Levitt, JSJ decompositions: definitions, existence, uniqueness. I: The JSJ deformation space arXiv:0911.3173v2

[17] F Haglund, D T Wise, Special cube complexes, Geom. Funct. Anal. 17 (2008) 15511620 MR2377497

[18] M Hall, Jr, Subgroups of finite index in free groups, Canadian J. Math. 1 (1949) 187-190 MR0028836 
[19] T Hsu, D T Wise, Cubulating graphs of free groups with cyclic edge groups, Amer. J. Math. 132 (2010) 1153-1188 MR2732342

[20] J Kahn, V Markovic, Immersing almost geodesic surfaces in a closed hyperbolic three manifold, to appear in Ann. of Math. arXiv:0910.5501

[21] O Kharlampovich, A Myasnikov, Irreducible affine varieties over a free group: I. Irreducibility of quadratic equations and Nullstellensatz, J. Algebra 200 (1998) 472-516 MR1610660

[22] S-h Kim, On right-angled Artin groups without surface subgroups, Groups Geom. Dyn. 4 (2010) 275-307 MR2595093

[23] S-h Kim, H Wilton, Polygonal words in free groups, to appear in Q. J. Math.

[24] L Louder, Scott complexity and adjoining roots to finitely generated groups, to appear in Groups Geom. Dyn.

[25] R C Lyndon, P E Schupp, Combinatorial group theory, Ergeb. Math. Grenz. 89, Springer, Berlin (1977) MR0577064

[26] J F Manning, Virtually geometric words and Whitehead's algorithm, Math. Res. Lett. 17 (2010) 917-925 MR2727618

[27] P Scott, Finitely generated 3-manifold groups are finitely presented, J. London Math. Soc. 6 (1973) 437-440 MR0380763

[28] P Scott, T Wall, Topological methods in group theory, from: "Homological group theory (Proc. Sympos., Durham, 1977)”, (C T C Wall, editor), London Math. Soc. Lecture Note Ser. 36, Cambridge Univ. Press (1979) 137-203 MR564422

[29] Z Sela, Diophantine geometry over groups I: Makanin-Razborov diagrams, Publ. Math. Inst. Hautes Études Sci. (2001) 31-105 MR1863735

[30] A Shenitzer, Decomposition of a group with a single defining relation into a free product, Proc. Amer. Math. Soc. 6 (1955) 273-279 MR0069174

[31] H Wilton, Elementarily free groups are subgroup separable, Proc. Lond. Math. Soc. 95 (2007) 473-496 MR2352568

[32] H Wilton, Hall's theorem for limit groups, Geom. Funct. Anal. 18 (2008) 271-303 MR2399104

[33] D T Wise, Subgroup separability of graphs of free groups with cyclic edge groups, Q. J. Math. 51 (2000) 107-129 MR1760573

Department of Mathematics, University College London

Gower Street, London, WC1E 6BT, UK

hwilton@math.ucl.ac.uk

http://www.homepages.ucl.ac.uk/ ucahhjr/

Proposed: Danny Calegari

Seconded: Martin R Bridson, Cameron Gordon

Received: 28 February 2011 Revised: 1 March 2011 